\title{
Warum es ohne Kriminalität nicht geht.
}

\author{
Wir brauchen Kriminalität und müssen sie doch zugleich verhindern: \\ Ein modernes Paradoxon?
}

Hermann Strasser und Henning van den Brink

\begin{abstract}
riminalität löst bei jedem von uns negative Assoziationen aus und taucht in der öffentlichen Diskussion ausschließlich als Problem auf, das bekämpft und bestraft werden muss - und am besten gar nicht erst entsteht. Als Ergänzung zu den polizeilichen Mitteln der Kriminalitätsbekämpfung durch Strafverfolgung und Gefahrenabwehr hat sich die kommunale Kriminalprävention inzwischen zum Leitbild für eine neue Sicherheitsordnung gemausert. Die Forderung, dass Prävention eine gesamtgesellschaftliche Aufgabe sei, ist mittlerweile eine gern zitierte Konsensformel. Ist Prävention endlich die Erlösung von der Sisyphosarbeit des ewigen Reagierens, wie es Manfred Kappeler (2000: 23) provokativ formuliert?
\end{abstract}

In den Städten und Gemeinden wird seit einigen Jahren eifrig »drauflos präveniert«: Kriminalpräventive Räte werden gegründet, Ordnungspartnerschaften eingerichtet, Gefahrenabwehrverordnungen erlassen, private Sicherheitsdienste engagiert, Videoüberwachungskameras aufgestellt, Bürger auf Streife geschickt. Eine Präventionskultur breitet sich aus, die unter verschiedenen Vorzeichen im öffentlichen Raum operiert. Zwischen mobiler Jugendarbeit und Mitternachtsbasketball einerseits und Null-Toleranz und Aufenthaltsverboten für Obdachlose andererseits bewegt sich der Präventionsdiskurs. Prävention wird beliebiger und erfreut sich zunehmender Beliebtheit bei Polizei und Verwaltung, Politik und Bürgerschaft. Denn der Präventionsdiskurs lehnt sich einmal an »law and order « an, grenzt sich dann aber wieder davon ab (vgl. Berner/Groenemeyer 2000: 107). Eckart Riehle (1988: 129) bemerkt dazu richtig, dass dem Begriff der Prävention eine selbst legitimierende Eigenschaft anhaftet - wer will schon verneinen, dass es besser ist zu handeln, bevor das Kind in den Brunnen gefallen ist?

Aber ist Kriminalität wirklich so schlecht für unsere Gesellschaft, so giftig für das Zusammenleben der Menschen? Diese Frage wagt kaum jemand zu stellen, sähe man sich doch nicht nur der Kritik der offiziellen Ordnungshüter und selbsternannten Moralapostel, sondern auch den erzürnten Gemütern gegenüber, die schon einmal Opfer eines Verbrechens geworden sind. Doch es geht bei dieser Frage nicht um Individuen, um Täter und Opfer von Kriminalität. Es geht vielmehr um die gesellschaftliche Funktion von Kriminalität.

Der französische Soziologe Emile Durkheim hat sich schon Ende des 19. Jahrhunderts dazu Gedanken gemacht. Durkheim hielt Kriminalität für normal, ja geradezu notwendig für die gesellschaftliche Entwicklung. Kleinkriminelle und Kapitalverbrecher, so seine These, seien keine assimilierten Fremdkörper in der Gesellschaft, sondern reguläre Tatsachen des sozialen Lebens (Durkheim 1976: 161).

Er begründet das damit, dass Verhaltensregeln die gesellschaftliche Ordnung stabil hielten und so Gesellschaft überhaupt erst möglich machten. Die Grenzen, die die Gesellschaft ihren Mitgliedern setzt, müssen aber immer wieder von Neuem öffentlich demonstriert werden. Und genau das geschieht durch Kriminalität, weil sie Grenzen überschreitet und Tabus bricht. Sie ist der Auslöser für Sanktionen, mit deren Hilfe gesellschaftliche Normen symbolisch verdeutlicht und ihre Geltung bewusst gemacht werden. An der Härte der Sanktion offenbart sich die Bedeutung der Regel für die Gesellschaft. Die Bestrafung sorgt dafür, dass die Norm, die durch die kriminelle Handlung verletzt worden ist, gleichsam »sichtbar« bleibt. »Die Definition dessen, was innerhalb der Gruppe als normal gilt, " so brachte es der amerikanische Soziologe Lewis A. Coser (1979: 24) auf den Punkt, »erfolgt also in Bezug auf das, was als abweichend angesehen wird; der Inhalt der Moral wird angegeben durch das, was nicht erlaubt ist.«

Über die Funktion der Normverdeutlichung hinaus lässt Kriminalität die Mitglieder der modernen Gesellschaft, die sonst divergierende Interessen verfolgen, zusammenrücken gegen jene, die die gesellschaftlichen Regeln nicht einhalten, betont neben Emile Durkheim auch George Herbert Mead (1918: 591). Die kollektive Bestrafung »im Namen des Volkes« vermittle den »anständigen Bürgern « das Gefühl von Solidarität und trage zur sozialen Kohäsion bei. Wo die soziale Kohäsion gering ist - so könnte man im Umkehrschluss sagen -, wird zu wenig gestraft und deswegen als eine Art gesellschaftlicher Selbstschutzmechanismus der Ruf aus der Bevölkerung nach mehr und härteren Strafen laut (vgl. Suhling et al. 2002: 174f.). Die Durkheimsche Theorie könnte so auch eine Erklärung für die steigende Punitivität in Staaten wie den USA liefern, wo sich ein hohes Maß an sozialer Ungleichheit mit einem geringen $\mathrm{Maß}$ an sozialer Kompensation und ökonomischer Intervention seitens des Staates verbinden.

Doch damit nicht genug. Verbrechen und Strafe stiften nicht nur Solidarität, verdeutlichen und konservieren die Normen, sondern verändern sie auch. So wahren sie die Anpassungsfähigkeit der Normen an den gesellschaftlichen Wandel, der immer auch ein moralischer Wandel ist. Ob es nun um die Unabhängigkeit des Denkens von Sokrates, die Forderungen nach gleicher Behandlung von Weißen und Schwarzen durch Martin Luther King, der Ruf nach dem Frauenwahlrecht oder der Selbstbestimmung über den eigenen Körper geht: Überall war das, was einmal als Verbrechen oder zumindest als Abweichung angesehen und sanktioniert wurde, der erste Schritt, das Vorspiel für überfällige Reformen von Traditionen, die nicht mehr mit den aktuellen Existenzbedingungen der Menschen übereinstimmten (vgl. Durkheim 1976: 160). Das Verbrechen von heute ist nicht selten die Moral von morgen. Das schließt freilich auch den umgekehrten Weg nicht aus, wenn wir an die Schmiergelder denken, die noch bis vor kurzem bei Auslandsaufträgen steuerlich absetzbar waren - und heute unter den Korruptionsparagraphen fallen. Wie sähe unsere Gesellschaft heute aus, wenn es nie »kriminelle« Geister wie Sokrates und Jesus von Nazareth oder »abweichendes Verhalten « wie die Musik der Beatles oder die Kunst von Andy Warhol gegeben hätte?

Warum also sollten wir all diese wichtigen Funktionen für unsere Gesellschaft unterdrücken, indem wir Kriminalität durch Kriminalprävention schon im Keim ersticken? Können wir nicht mit Durkheim die Gleichung aufstellen Kriminalität = funktional, Kriminalprävention = dysfunktional? Die Antwort lautet: Nein. Wie Kriminalität nimmt auch Prävention im Lichte ihrer gesellschaftlichen Funktionalität eine eigene Gestalt an. Kriminalprävention ist - um die eingangs provozierten Gemüter zu beruhigen ebenso wenig kontraproduktiv oder überflüssig wie Kriminalität. 
Damit Kriminalität nämlich ihre wichtigen Funktionen erfüllen kann, muss sie entdeckt und bestraft, muss ein Übeltäter gefunden und aus dem viel zitierten Dunkelfeld ans Tageslicht gezerrt werden. Es reicht nicht aus, dass Kriminalität einfach nur existiert. Weil die Entdeckung und Aufklärung von Straftaten aber zu 90 Prozent durch die Anzeige von Bürgern stattfindet, die die Täter häufig gleich "mitliefern", zählt die Erhöhung der Anzeigebereitschaft zu den vorrangigen Aufgaben der polizeilichen Präventionsarbeit. Das ist z.B. bei jungen Russlanddeutschen gar nicht so leicht. Für Viele von ih nen ist das Schweigen über eine Straftat eine Frage der Ehre (Strasser/Zdun 2003). Die in solchen subkulturellen Milieus hoffähig gemachte Selbstjustiz ist nicht nur im Hinblick auf Moral, Legitimation und Gerechtigkeit, sondern auch im Hinblick auf die Funktionalität verwerflich, weil sie im Hinterhof und nicht auf der Vorderbühne der Gesellschaft vollzogen wird. Aber nur dort kann die staatliche Maschinerie der öffentlichkeitswirksamen Normverdeutlichung ins Rollen kommen. Und nur so können Polizei, Justiz und Strafvollzug ihre Handlungsfähigkeit demonstrieren und Rechtsvertrauen schaffen.

Kriminalpräventive Maßnahmen können außerdem eine breitere Aufmerksamkeit auf sich ziehen, als die meisten Strafrechtsurteile das vermögen. Ihr Stadt(teil)bezug und ihre konkrete Greifbarkeit besitzen einen hohen Nachrichtenwert für die Lokalpresse, was ihnen wiederum die Unterstützung von Kommunalpolitikern sichert und damit zusätzliches Gewicht verleiht. Während präventive Maßnahmen und Konzepte z.B. in kommunalen Präventionsgremien in Kooperation mit betroffenen und beteiligten Akteuren aus Politik, Verwaltung, Polizei, Schulen, Gewerkschaften, Einzelhandel, Kirchen, Vereinen und Verbänden entwickelt und umgesetzt werden und in ein kommunales Umfeld eingebettet sind (vgl. Berner/Groenemeyer 2000; van den Brink 2005), hat sich das Justizsystem im Zuge seiner Ausdifferenzierung und Professionalisierung immer stärker von dem Rest der Gesellschaft abgekapselt. Prävention schafft also einen zusätzlichen »Kommunikationskanal «, der das Rechtsbewusstsein der Gesellschaftsmitglieder zu schärfen vermag (Bussmann 2000: 6).

In der heutigen Mediengesellschaft, in der die Bürger durch die Multiplikatoren Fernsehen, Radio und Zeitung immer mehr über Kriminalität erfahren, die um sie herum passiert, müssen sie auch immer mehr erfahren, dass etwas dagegen unternommen werde, wenn nicht ihr Vertrauen in den Rechtsstaat schwinden soll. Die häufig zu beobachtende Umbenennung von bestehenden sozialen Projekten in Präventionsprojekte und deren kommunalpolitische Aufwertung erscheint durch die Durkheimsche Brille gesehen durchaus funktional. Mit dem gleichen Blickwinkel kann man sogar der oft kritisierten Konzentration kriminalpräventiver Projekte auf Bagatell- und Ju- gendkriminalität positive Seiten abgewinnen. Dadurch gelangen nämlich nicht nur Verstöße gegen elementare Normen wie Mord, sondern auch Normverletzungen geringeren Unrechtgehalts wie Graffiti, Sachbeschädigung oder Ladendiebstahl ins öffentliche Bewusstsein. Und das verhindert ein allmähliches Erodieren des Normbestands von seinen Rändern her.

Kriminalprävention ist nicht zuletzt ein soziales Bindemittel, weil sie ein sinn- und identitätsstiftendes Orientierungsgut für eine neue Gemeinschaftlichkeit zur Verfügung stellt (Frehsee 1998: 751). Über den Umweg der Kriminalprävention kommt es im sozialen Nahraum zu einem Bündnis gegen den »gemeinsamen Feind «, der Kriminalität heißt. Schließlich will niemand Opfer eines Verbrechens werden. Darin sind sich alle einig - trotz oder vielleicht gerade wegen der fortschreitenden Pluralisierung der Lebensstile und Individualisierung der Lebensläufe.

Die mit diesen Schlagworten belegten Umwälzungen in den gesellschaftlichen Teilsystemen schlagen auch auf das Recht durch. Die Risikogesellschaft (Beck 1986) bereitet den Weg für das Risikostrafrecht (Frehsee 1999: 16). Die Präventionskonjunktur ist ein Zeichen für die zunehmenden Anstrengungen und Erwartungen, Bedürfnisse und Erfordernisse, Schadenspotenziale jeder Art für Individuum und Gesellschaft beherrschbar und berechenbar zu machen (Hesse 1994: 187f.). In diesem Zusammenhang wird das Recht auf der einen Seite immer wichtiger, um Erwartungssicherheit und Vertrauen in den Rechtsstaat zu schaffen (Luhmann 1997). Auf der anderen Seite ist das Instrumentarium des Rechts ganz überwiegend auf tertiäre, postdeliktische Prävention festgelegt. Damit kann es aber die gestiegenen und übersteigerten Ansprüche freilich nicht mehr erfüllen. Das Strafurteil stellt eine reaktive Intervention dar, es ist keine proaktive Prävention. Das Recht trägt die Verantwortung für die Resozialisierung des verurteilten Straftäters, für die Sozialisation des potenziellen Straftäters sind andere Institutionen zuständig. Die Bearbeitung des Problems steigender Kriminalitätsfurcht kann ebenfalls nur zu einem kleinen Teil in den Gerichtsälen und Strafgefängnissen geleistet werden, denn ihre Hauptursachen liegen woanders. Richter und Staatsanwälte sind stets mit den Folgen von Kriminalität konfrontiert, die Bekämpfung der Ursachen von Kriminalität und Kriminalitätsfurcht ist aber Aufgabe der Politik und der Verwaltung. Die Notwendigkeit, dieses doppelte Dilemma systemischer Kompetenz zu überbrücken, dürfte ein wichtiger Antrieb dafür gewesen sein, dass sich Kriminalprävention als autonomes Subsystem inzwischen funktional weitgehend verselbständigt hat (vgl. Lehne 1998; Sack 1995). Kommunale Kriminalprävention hat die Leerstelle im Bereich der sekundären Prävention besetzt.
Doch keineswegs alles, was in den letzten Jahren an Präventionsmaßnahmen ergriffen worden ist, würde Durkheim gutheißen. Wenn beispielsweise aus "neighborhood programs « »gated communities« werden und der informelle $\mathrm{Zu}$ sammenschluss gegen Kriminalität $\mathrm{zu}$ einem räumlich manifestierten Ausschluss von Kriminalität mutiert, zerfällt über kurz oder lang die Gesellschaft, weil ohne die Wahrnehmung von Kriminalität auch ihre wichtigen Funktionen für deren Erhaltung und Weiterentwicklung ausbleiben. Ebenso kritisch ist die sich abzeichnende rechtsstaatliche Entgrenzung von Prävention zu sehen, die mit der offensiv-defensiven Forderung vorangetrieben wird, dass die Präventionsanstrengungen weiter verstärkt werden müssten, weil sie zur wirksamen Vermeidung von Kriminalität nicht (mehr) ausreichten (vgl. Frehsee 2000: 68; Kappeler 2000: 27). Vorläufiger Höhepunkt dieser Entwicklung war der »Präventivkrieg « der »Weltpolizei USA « gegen den »Schurkenstaat «Irak. Hier drängt sich die Frage auf, ob durch eine solche massive Ausdehnung der Legitimationsgrundlage für präventives Handeln die für die gesellschaftliche Entwicklung so wichtige Ausgewogenheit von Freiheit und Sicherheit ernsthaft in Gefahr gerät und ob sich Demokratien auch selbst zerstören können, wenn sie zur Bekämpfung des »Bösen « auf »böse« Mittel zurückgreifen (vgl. Ignatieff 2005).

Trotzdem hätte Emile Durkheim in der heutigen Präventionspraxis keinen Widerspruch zu seinen Thesen gesehen. Im Gegenteil: Kriminalität und Kriminalprävention ergänzen und verstärken sich in ihren Wirkungen wechselseitig und das nicht etwa zum Nachteil, sondern im Dienste der Gesellschaft. Wir brauchen also beides: Kriminalität, damit es weiterhin Strafen gibt, und Kriminalprävention, damit es weiterhin Kriminalität gibt.

\section{Literatur}

Beck, U. (1986): Risikogesellschaft. Auf dem Weg in eine andere Moderne, Frankfurt/Main: Suhrkamp.

Berner, F. und A. Groenemeyer (2000): »... denn sie wissen nicht, was sie tun « - Die Institutionalisierung kommunaler Kriminalprävention im Kriminalpräventiven Rat, Soziale Probleme 11 (Heft 1/2), S. 83-115.

Brink, H. van den (2005): Kommunale Kriminalprävention - Mehr Sicherheit in der Stadt? Eine qualitative Studie über kommunale Präventionsgremien, Frankfurt/Main: Verlag für Polizeiwissenschaft.

Bussmann, K.-D. (2000): Das Ei in der Backmischung. Funktionen kommunaler Kriminalprävention in einer modernen Gesellschaft, Manuskript zum Vortrag auf dem 19. Landespräventionstag in Magdeburg, 19. Oktober 2000, verfügbar unter: http://www.jura.uni-halle.de/download/bussmann/ publik01.pdf.

Coser, L.A. (1979): Einige Funktionen abweichenden Verhaltens und normative Flexibilität, in: F. Sack und R. König (Hrsg.), Kriminalsoziologie, 3., unveränderte Aufl., Wiesbaden: AVG, S. 21-37. 
Durkheim, E. (1976): Die Regeln der soziologischen Methode, R. König (Hrsg.), 5. Aufl., Darmstadt [u.a.]: Luchterhand.

Frehsee, D. (1998): Politische Funktionen Kommunaler Kriminalprävention, in: H.-J. Albrecht, F. Dünkel H.-J. Kerner, J. Kürzinger, H. Schöch, K. Sessar und B. Villmow (Hrsg.), Internationale Perspektiven in Kriminologie und Strafrecht - Festschrift für Günther Kaiser zum 70. Geburtstag, Berlin: Duncker \& Humblot, S. 739-763.

Frehsee, D. (1999): Verschwindet der Rechtsstaat?, in: Neue Kriminalpolitik, 11 (Heft 1), S. 16-21.

Frehsee, D. (2000): Entstrukturierung und Extensivierung kriminalistischer Verhaltenskontrolle, in: $\mathrm{H}$ Rottleuthner (Hrsg.), Armer Rechtsstaat, Baden-Baden: Nomos, S. 53-74.

Hesse, H.A. (1994): Der Schutzstaat. Rechtssoziologische Skizzen in dunkler Zeit, Baden-Baden: Nomos.

Ignatieff, M. (2005): Das kleinere Übel. Politische Moral in einem Zeitalter des Terrors, Berlin: Philo.

Kappeler, M. (2000): Prävention als Fetisch (in) der Jugendhilfe, Neue Kriminalpolitik 12 (Heft 2), S. 23-27.
Lehne, W. (1998): Kommunale Kriminalprävention. Die Reorganisation des Politikfeldes »Innere Sicherheit«, in: R. Hitzler und H. Peters (Hrsg.), Inszenierung: Innere Sicherheit, Daten und Diskurse, Opladen: Leske + Budrich, S. 113-130.

Luhmann, N. (1997): Das Recht der Gesellschaft, Frankfurt/Main: Suhrkamp.

Mead, G.H. (1918): The Psychology of Punitive Justice, American Journal of Sociology 23 (Heft 5), S. 577-602.

Riehle, E. (1988): Von der repressiven zur präventiven Polizei - oder: Die Verpolizeilichung der Prävention, in: R. Appel; D. Hummel und W. Hippe (Hrsg.), Die neue Sicherheit. Vom Notstand zur Sozialen Kontrolle, Köln: Kölner Volksblatt, S. 129-139.

Sack, F. (1995): Prävention - ein alter Gedanke in neuem Gewand. Zur Entwicklung und Kritik der Strukturen »postmoderner « Kontrolle, in: R. Gössner (Hrsg.), Mythos Sicherheit. Der hilflose Schrei nach dem starken Staat, Baden-Baden: Nomos, S. 429-456.

Strasser, H. und S. Zdun (2003): Ehrenwerte Männer Jugendliche Russlanddeutsche und die deutsche Polizei, Zeitschrift für Jugendkriminalrecht und Jugendhilfe 1 (Heft 3), S. 266-271.
Suhling, S., T. Schott und K. Brettfeld (2002): Steigen de Gefangenenzahlen - Probleme und mögliche Ursachen, Soziale Probleme 13 (Heft 2), S. 156-184.

\section{Anmerkungen}

Es handelt sich bei diesem Beitrag um eine stark gekürzte und veränderte Version eines Aufsatzes, der kürzlich in der Zeitschrift für Rechtssoziologie (Heft 2/2004) erschienen ist. Für hilfreiche Anregungen zur Überarbeitung dieses Beitrags bedanken wir uns herzlich bei Prof. Dr. Monika Frommel.

Hermann Strasser ist Professor für Soziologie an der Universität Duisburg-Essen, Henning van den Brink ist derzeit Promotionsstudent an der Universität Münster und wissenschaftliche Hilfskraft an der Universität Köln.

\section{REZENSIONEN}

\section{Alexander Vollbach}

Historische Studien zur Verbrechensbekämpfung

Ziel der sozial- und verwaltungsgeschichtlichen Studie ist es, die Wechselbeziehungen zwischen Psychiatrie, Kriminologie und Strafrechtswesen über einen Untersuchungszeitraum von 6 Jahrzehnten (1871-1933) zu erforschen. Im Vordergrund der Studie stehen die wissenschaftlichen, politischen und kulturellen Diskurse über den »verbrecherischen Menschen «.

Thematisiert werden das Engagement der Forensischen Psychiatrie im Rahmen der Gutachtertätigkeit und medizinischen »Verbrecherlehre«, die Unterbringungspraxis geisteskranker Rechtsbrecher in Irren- und Strafanstalten, kriminalpolitische Forderungen der Forensischen Psychiatrie und die Reaktion der Strafrechtswissenschaft sowie der Justizverwaltung. Die Auswirkungen zeithistorischer bzw. politischer Einschnitte - hier der Erste Weltkrieg - auf die kulturelle Wahrnehmung von Geisteskrankheit und Kriminalität werden ebenfalls erörtert.

Der Autor wertet dazu den Nachlass des Anstaltsstaates aus, also die Archivbestände von Landschaftsverbänden als Träger der Anstaltspsychiatrie, die Archive der Regierungsbezirksund Oberlandesgerichtsbezirke sowie der Archiv-bestand der Länder. Die alltagsgeschichtliche Betrachtung der Lebenswelt des Verbrechers im Anstaltsstaat, das Anstaltsleben mit seiner »konstanten Repression « (S. 19) wird dagegen ausgespart, ebenso thematische Nachbargebiete wie die religiös motivierte Gefängnisreform des 19. Jahrhunderts oder die Entwicklung des Jugendstrafrechts.

Für Müller wurde der »Krieg gegen das Verbrechertum [...] mit allen Waffen geführt, die der moderne Anstaltsstaat bereit hielt: Gesetze und Anstalten, Wissenschaften und Behörden, Fachbeamte, Geheimräte, SachverständigenGutachten, - und wenn diese versagten - in zweiter Linie: Verwaltungsvorschriften und Spezialanstalten, Forschungsförderung und Sonderbehörden, Beamten-Schulungen, Expertengremien, Obergutachten.«(S. 298).

Das »Kompetenzchaos [...] einer auf Bürokratie, Fachwissen und Fürsorge gestützten Verbrechensbekämpfung « bei Fragen der Behandlung von kriminellen führte zu einem strategischen Wechsel: im Zuge einer allgemein gestiegenen Wertschätzung der modernen Psychiatrie sowie einer allgemeinen Wissenschaftsgläubigkeit sollten im Strafrecht anstelle der Grundprämissen des schuldbasierten Vergeltungsstrafrechts nach dem Willen der Strafrechtsreformer die »Gefährlichkeit« und »Besserungsfähigkeit« des Täters ausschlaggebend sein (S. 299). Die Verwissenschaftlichung der Kriminalpolitik sei dem Autor zufolge allerdings dem »Grundmuster eines dem tatsächlichen Wissensstand vorauseilenden Szientismus« (S. 300) gefolgt. Die Selbstermächtigung und Selbstüberschätzung der Psychiater, die hinter der Verwissenschaftlichung und Rationalisierung der Strafrechtspflege steckte, entsprach laut Müller nicht dem tatsächlichen Wissenschaftsfortschritt: »Die Wissenschaftsgläubigkeit, nicht der wissenschaftliche Fortschritt war die treibende Kraft hinter der >Rationalisierung < des Strafens." (S. 299).

Die Reformpolitik der Weimarer Republik gilt als Experimentierfeld der Kriminalpolitik. Müller erörtert die Klassifizierung der Strafgefangenen im bayerischen Stufenstrafvollzug durch den Kriminalbiologischen Dienst (namentlich Theodor Viernstein), der in Bayern eng mit Deutschen Forschungsanstalt für Psychiatrie in München (Ernst Rüdin) kooperierte. Auf die sich daraus ergebende Etablierung der Kriminalbiologie als Wissenschaft wird ebenfalls eingegangen wie auf deren Fehlentwicklung. Seit der Kaiserzeit, insbesondere aber am Ende der Weimarer Republik eskalierte die Verbrechensbekämpfung stufenweise und nahm im Nationalsozialismus verbrecherische Züge an (S. 273 ff.).

Müller thematisiert vor allem die politische Überformung der Strafrechtsreformpläne im Zuge der Gesetzgebungsverfahren. Er kommt zu dem Ergebnis, dass »die Geschichte der Strafrechtsreform reich an Beispielen dafür [ist], dass >moderne Reformen ausgebremst, politisch instrumentalisiert und schließlich rassistisch umgebogen wurden. Gerade die wiederholten Verschärfungen, welche die Gesetzentwürfe in einem Autorität geprägten politischen Umfeld bis 1933 erfahren hatten, bereiteten der inhumanen Rechtssprechungspraxis im Dritten Reich den Weg.« (S. 298).

Müller versteht seine Studie als Beitrag zur Medizingeschichte, die an neuere sozialgeschichtliche Arbeiten zur Expansion medizinischer Deutungsmacht (»Medikalisierung«) anknüpft. Mit der Erforschung der »Schnittflächen zwischen psychiatrisch-kriminologischer Wissenschaft und der Praxis des Strafsystems « hat Müller einen Gegenstandsbereich in den historischen Blick genommen, dem bisher wenig Beachtung geschenkt wurde. Die gut zu lesende und spannende Studie schließt mit einem umfangreichen Quellen- und Literaturverzeichnis.

Christian Müller:

Verbrechensbekämpfung im Anstaltsstaat. Psychiatrie,

Kriminologie und Strafrechtsreform in Deutschland 1871-1933 [Kritische Studien zur Geschichtswissenschaft Band 160], Vandenhoeck

Ruprecht. Göttingen 2004, 337 Seiten

Der Autor ist Kriminologe und am Niedersächsischen Landeskrankenhaus Moringen tätig. 